\title{
GABA agonists truly helping sleep or is it just a dream?
}

\author{
María Robles-Martínez ${ }^{1 *}$ and Gustavo Añez Sturchio ${ }^{2}$ \\ ${ }^{1}$ Department of Psychiatry, Addiction and dual Diagnosis Unit, Vall d' Hebron University Hospital, Passeig Vall d'Hebron, 119-129, Barcelon-08035, Spain \\ ${ }^{2}$ Department of Rheumatology, Vall d' Hebron University Hospital, Passeig Vall d'Hebron, 119-129, Barcelon-08035, Spain
}

\begin{abstract}
We present a commentary in order to reflect about the use of GABA agonists in clinical practice to treat sleep disorders. Noticing that drugs like GABA agonists are used frequently as alternatives in patients with sleep disturbances and respiratory problems as well as in fibromyalgia patients where is also common to see respiratory disorders with truly few objectified tests on respiratory parameters, we believe more studies must be done in the matter and will like to publish the article in the journal to raise awareness and make clinical professionals reflect about the use of polysomnography for such purpose. The fact that GABA agonists can cause an increase of the apnea-hypopnea index as it is recently being discussed is also important to be aware of. We also highlight the fact that it is worth studying if this drugs could cause obstructive sleep apnea by themselves and also that sleep disturbances must be studied and looked at before attributing them to psychiatric causes.
\end{abstract}

\section{Commentary}

In clinical psychiatry it is usual to daily encounter patients that suffer from insomnia that demand hypnotic medication. It's also common for our patients to have serious respiratory problems, due to nicotine dependence among other factors that contraindicate the use of hypnotic medications or benzodiazepines. It is also a common practice in clinical rheumatology to use neuroleptic and hypnotic medication to aid pain in fibromyalgia patients where it is frequent to see obesity and associated obstructive sleep apnea (OSA). Under these situations it is a common practice to use alternative drugs like GABA agonists such as pregabalin or gabapentin.

As clinical professionals we have found that few studies truly focus on how to increase sleep quality in patients with fibromyalgia. This is important to look at since a relation can be made between the patient's pain and poor quality of sleep and not many studies have been done with objective tests [1]. GABA agonists are a common management strategy used in fibromyalgia inspired by such theory. It has recently been found though that the use of $300 \mathrm{mg}$ daily of gabapentin in healthy adult men increases the risk of OSA due to raises in the apnea-hypopnea index (AHI) and oxygen desaturation index (ODI) seen by polysomnography [2]. It is also evidenced that these negative effects during sleep are also superior to the ones seen in studies with flurazepam [3] and antipsychotic medications [4].

Despite this, there are other studies that recognize the beneficial effect of gabapentin in insomnia and fibromyalgia [5-7] without reporting significant adverse effects, yet these studies did not perform a polysomnography. This could be quite troublesome since it is common to see respiratory problems during sleep in fibromyalgia patients $[8,9]$. It's why we believe that it is convenient to confirm the beneficial effects of sleep medications with complementary tests that objectify not only their benefit but also the possible adverse effects that could have repercussions in sleep and other parameters that influence sleep, like AHI.

It is also important to differentiate between the subjective quality of sleep referred by the patient and proven objectified quality of sleep by an external observer since this could influence diagnostic orientation and treatment. This is especially important in patients with anxiety and symptoms of depression as it has recently been published that the sleep cycle plays a role in emotion regulation and sleep disturbances may play a role in the emotion dysregulation observed in anxiety and depressive disorders [10]. These pathologies also can favor abuse of such medication which is also an issue that must be taken into account

We also highlight that currently, systematic revisions to understand the effects of pregabalin and gabapentin in the treatment of bipolar disorder, insomnia and anxiety are taking place [11], but it seems that polysomnographic parameters are not being taken into account which as the systematic review states are also important co-morbid disorders in fibromyalgia patients.

We consider that these type of studies are of upmost importance in daily clinical practice and their dissemination among professionals that treat patients with sleep disorders must be mandatory since it is very common to see prescription of these type of drugs in patients with sleep disorders and unknowingly we can actually be favoring the development of respiratory problems that can affect bed rest and overall wellbeing being more of a nightmare than a favorable dream.

\section{References}

1. Keskindag B and Karaaziz M (2017) The association between pain and sleep in fibromyalgia. Saudi Med J 38: 465-475. [Crossref]

2. Piovezan RD, Kase C, Moizinho R, Tufik S, Poyares D (2017) Gabapentin acutely increases the apnea-hypopnea index in older men: data from a randomized, doubleblind, placebo-controlled study. J Sleep Res 26: 166-170. [Crossref]

3. Dolly FR, Block AJ (1982) Effect of flurazepam on sleep-disordered breathing and nocturnal oxygen desaturation in asymptomatic subjects. Am J Med 73: 239-243. [Crossref]

Correspondence to: María Robles-Martínez, Department of Psychiatry, Addiction and dual Diagnosis Unit, Vall d' Hebron University Hospital, Passeig Vall d'Hebron, 119-129, Barcelon-08035, Spain, Tel: +34934893880; E-mail: correo@mariarobles.es

Key words: sleep, GABA agonists, gabapentin, fibromyalgia

Received: June 09, 2017; Accepted: June 27, 2017; Published: June 29, 2017 
4. Rishi MA, Shetty M, Wolff A, Amoateng-Adjepong Y, Manthous CA (2010) Atypical antipsychotic medications are independently associated with severe obstructive sleep apnea. Clin Neuropharmacol 33: 109-113. [Crossref]

5. Cho YW, Song ML (2014) Effects of pregabalin in patients with hypnotic-dependent insomnia. J Clin Sleep Med 10: 545-550. [Crossref]

6. Furey SA, Hull SG, Leibowitz MT, Jayawardena S, Roth T (2014) A randomized, double-blind, placebo-controlled, multicenter, 28- day, polysomnographic study of gabapentin in transient insomnia induced by sleep phase advance. J Clin Sleep Med 10: 1101-1109. [Crossref]

7. Moore RA, Philip JW, Sheena D, Andrew SC (2014) Gabapentin for chronic neuropathic pain and fibromyalgia in adults. Cochrane Database Syst Rev: CD007938. [Crossref]
8. Marvisi M, Balzarini L, Mancini C, Ramponi S, Marvisi C (2015) Fibromyalgia is frequent in obstructive sleep apnea and responds to CPAP therapy. Eur J Intern Med 26: 49-50. [Crossref]

9. Martinez D, Cassol CM (2008) Fibromyalgia and sleep-disordered breathing: the missing link. Arthritis Res Ther 10: 408. [Crossref]

10. Klumpp H, Roberts J, Kapella M, Kennedy AE, Kumar A, et al. (2017) Subjective and objective sleep quality modulate emotion regulatory brain function in anxiety and depression. Depress Anxiety : 1-10. [Crossref]

11. Houghton KT, Forrest A, Awad A, Atkinson LZ, Stockton S, et al. (2017) Biologica rationale and potential clinical use of gabapentin and pregabalin in bipolar disorder, insomnia and anxiety: protocol for a systematic review and meta-analysis. BMJ Open 7: e013433. [Crossref]

Copyright: (C2017 Robles-Martínez M. This is an open-access article distributed under the terms of the Creative Commons Attribution License, which permits unrestricted use, distribution, and reproduction in any medium, provided the original author and source are credited. 